\title{
Combinación de cuestionarios simples y gamificados utilizando gestores de participación en el aula: experiencia y percepción del alumnado*
}

\author{
Sandra Roger ${ }^{1}$, Maximo Cobos $^{1}$, Miguel Arevalillo-Herráez ${ }^{1}$ y Miguel \\ García-Pineda $^{1}$ \\ ${ }^{1}$ Departament d'Informàtica, Universitat de València
}

\begin{abstract}
The growing use of mobile devices has motivated the development of a wide range of applications to help manage the students' participation in the classroom. Socrative allows the lecturer to use multiple-choice questionnaires in the classroom, either in a simple or a gamified mode (Space Race). In this paper, we describe our experience at using this tool to promote competitive learning, at both undergraduate and post-graduate levels. The student's perception indicates that the use of the application helped at increasing engagement and motivation. However, relevant differences were found between both modes of use, underlining the importance of an adequate activity design.
\end{abstract}

Keywords: gamification, student response system, Socrative.

\section{Resumen}

En los últimos años y gracias a la utilización masiva de dispositivos móviles, han proliferado múltiples aplicaciones para la gestión de la participación del alumnado en el aula. Específicamente, Socrative, permite el lanzamiento por el profesor de cuestionarios de opción múltiple en modo simple o en modo gamificado (carrera espacial), fomentando asi el juego y la competitividad. En este trabajo se describe la experiencia obtenida mediante la combinación de ambos modos en una asignatura de Grado y otra de Máster. Los resultados obtenidos así como la percepción del alumnado indican que los alumnos se ven altamente motivados por este tipo de herramientas, si bien existen diferencias importantes en ambos modos de utilización que conviene tener presentes en el diseño de la actividad.

Keywords: gamificación, gestor de participación, Socrative. 


\section{Introducción}

La gamificación en la docencia presencial universitaria se ha convertido actualmente en un elemento clave de la innovación educativa (Kay y LeSage 2009, Melhuish y Falloon 2010). Son muchos los estudios que demuestran que la utilización de actividades lúdicas en el aula es un recurso muy efectivo para mejorar la implicación de los estudiantes en el proceso de enseñanza-aprendizaje (Benítez-Porres 2015, Frías, Arce y Flores-Morales 2016). El establecimiento de reglas de juego y la recompensación con puntos, niveles o rankings, consigue formentar la autoafirmación del estudiante, su esfuerzo personal y la colaboración con el resto de sus compañeros. Además, las estrategias de gamificación pueden ser muy diversas, ayudando no sólo al afianzamiento de los conocimientos relativos a los contenidos impartidos, sino también al desarrollo personal y social del alumnado.

Por otro lado, la introducción de herramientas digitales y el uso masivo de dispositivos móviles en la aulas han llevado a un importante cambio tecnológico que ha desembocado en la aparición de nuevas herramientas en línea y aplicaciones que tienen como objetivo introducir elementos innovadores en las clases (Eisele-Dyrli 2011). Algunas de estas herramientas no sólo incorporan funcionalidades de respuesta de audiencia, sino que también implementan modos de utilización que fomentan el uso de estrategias gamificadoras (Calvillo y Martín 2017). Si bien la forma en la que estas aplicaciones pueden ser utilizadas pueden variar de un contexto docente a otro, es importante dar el peso adecuado y adaptar su uso de acuerdo al contenido y a las consideraciones pedagógicas del contexto de aprendizaje específico.

Entre las herramientas de gestión de la participación que más popularidad han adquirido en los últimos años se encuentran Kahoot y Socrative (Fuertes y col. 2016). Estas herramientas pueden ser utilizadas en aulas de tamaños muy variados y de cualquier nivel educativo. El tipo de respuesta que recoge el sistema viene determinado por el diseño de la actividad, siendo el cuestionario de múltiple opción el más habitual. A medida que los estudiantes van respondiendo, el profesor puede ir descubriendo las respuestas correctas, obteniendo una estadística instantánea del grado de acierto conseguido por la clase. Si bien ambas herramientas son excelentes y tienen una funcionalidad similar, Socrative introduce un modo de participación especialmente diseñado para fomentar la gamificación, la llamada carrera espacial (Space Race). Este modo divide a los alumnos por grupos en cohetes de distinto color, los cuales avanzan en función de las respuestas correctas observadas.

En este artículo valoramos la experiencia obtenida a través de la combinación de estos dos modos de utilización de Socrative: el llamado modo simple (lanzamiento de cuestionarios de opción múltiple) y el modo gamificado de carrera espacial (juego de competición). Ambos modos han sido utilizados en dos asignaturas de la Escola Tècnica Superior d'Enginyeria de la Universitat de València, una de ellas perteneciente al Grado de Ingeniería Telemática y otra al Máster Universitario de Ingeniería de Telecomunicación. En la experiencia analizamos tres aspectos que consideramos importantes para plantear estrategias de evaluación futuras basadas en estas herramientas:

- Impacto que ambos modos de operación tienen en la motivación del alumnado. 
- Grado de preparación por parte de los estudiantes para cada tipo de cuestionario.

- Diferencia en las calificaciones obtenidas por los alumnos bajo ambos modos.

Para evaluar los anteriores aspectos, se tuvieron en cuenta tanto los informes de resultados proporcionados por Socrative correspondientes a los cuestionarios técnicos, como los resultados de encuestas que los alumnos rellenaron al final del curso (también mediante Socrative). Los resultados muestran que, si bien la motivación y la implicación en la actividad son mayores en el modo gamificado, las calificaciones obtenidas son menores que en el caso del modo simple.

El artículo está estructurado en las siguientes secciones. La sección 2 describe brevemente las herramientas de gestión de participación y los modos de operación utilizados en este trabajo. La sección 3 describe la metodología seguida en el mismo. La sección 4 discute los resultados obtenidos a partir de la utilización de las herramientas y los cuestionarios. Finalmente, la sección 5 resume las conclusiones extraídas de esta experiencia.

\section{Gestores de participación}

Los gestores de participación o herramientas de respuesta de audiencia como Kahoot (Kahoot s.f.) o Socrative (Socrative s.f.) se basan en un sistema inteligente de respuesta a través de Internet, accediendo a través de un dispositivo conectado, ya sea un ordenador, un teléfono inteligente o una tableta. El diseño de la actividad lo realiza el profesor, controlando el flujo de preguntas a su elección. El acceso a la actividad por los alumnos es muy sencillo, consistiendo únicamente en una identificación (en algunos casos sin registro previo) que les permite interactuar con el contenido de la actividad de forma inmediata. El tipo de respuesta que recoge el sistema viene determinado por el diseño de la actividad, siendo el cuestionario de múltiple opción el más habitual. A medida que los estudiantes van respondiendo, el profesor puede ir descubriendo las respuestas correctas, obteniendo una estadística instantánea del grado de acierto conseguido por la clase. Una vez terminada la actividad, estas herramientas suelen ofrecer un informe donde se muestran las respuestas individuales proporcionadas por cada alumno, así como las estadísticas globales obtenidas en la misma. La principal ventaja de estas herramientas es que no existe la necesidad de disponer de aparatos electrónicos adicionales a los utilizados de forma habitual por los alumnos, siendo el único requisito tener algún tipo de dispositivo con conexión a Internet. Otra ventaja muy interesante es que los resultados de los cuestionarios no tienen por qué ser públicos, siendo únicamente conocidos por el profesor.

En este trabajo nos hemos centrado exclusivamente en la herramienta Socrative por ofrecer un modo especial diseñado para la gamificación. Describimos a continuación esta herramienta y los dos modos utilizados. 


\subsection{Socrative}

Socrative es un software de uso muy intuitivo que puede ser utilizado para diversas actividades, ofreciendo, entre otras, las siguientes funcionalidades:

- Obtención de feedback en tiempo real. El planteamiento de cuestiones al alumnado mediante Socrative permite de manera cómoda y eficiente obtener información sobre el seguimiento de la clase.

- Evaluación de conocimientos previos. Los quizzes o cuestionarios pueden ser utilizados con anterioridad o al principio de una clase para evaluar, por ejemplo, prerrequisitos de una asignatura, o para comprobar si los estudiantes han revisado algún material y lo han entendido antes de la clase.

- Accesibilidad a la participación. Realizar los cuestionarios mediante la aplicación favorece que todo el alumnado pueda tener accesible la participación, sean cuales sean sus capacidades de comunicación.

- Evaluación continua. Socrative permite conocer de forma reiterada a lo largo de la clase o del curso los conocimientos que se deben ir adquiriendo.

\subsection{Modos simple y gamificado}

Los dos modos de operación de Socrative utilizados en este trabajo son los siguientes:

1. Modo simple: Este modo consiste en la presentación de cuestionarios de opción múltiple al ritmo marcado por el profesor (Fig. 1). El profesor lanza el cuestionario al que acceden los alumnos mediante sus dispositivos, desvelando la respuesta a cada una de las preguntas cuando todos los estudiantes han seleccionado una respuesta. No existe limitación temporal, por lo que los alumnos disponen del tiempo que necesiten para seleccionar su respuesta. Al desvelar la opción correcta la puntuación se mantiene anónima, por lo que los alumnos no saben qué ha contestado cada compañero, sólo el porcentaje de acierto general.

2. Modo gamificado: Este modo hace uso de la opción "Space Race" (carrera espacial) de Socrative (Fig. 2). Los alumnos se organizan en equipos, con la opción de establecer los equipos de forma aleatoria. Cada equipo se representa por un cohete de un determinado color, que avanza siempre que un miembro del equipo acierta la respuesta correcta. Como en este trabajo se pretende comparar el rendimiento individual del alumno en ambos modos, se consideran tantos equipos como alumnos, por lo que la competición es individual y no a nivel de grupo. Aunque no existe cronómetro para responder cada pregunta, el alumno puede observar cómo avanzan el resto de compañeros, lo que crea cierta presión para responder.

El uso de dispositivos electrónicos, usados de manera habitual por el alumnado en la actualidad, proporciona una motivación extra para responder. Además, el modo gamificado favorece una competitividad sana con uno mismo para mejorar. 


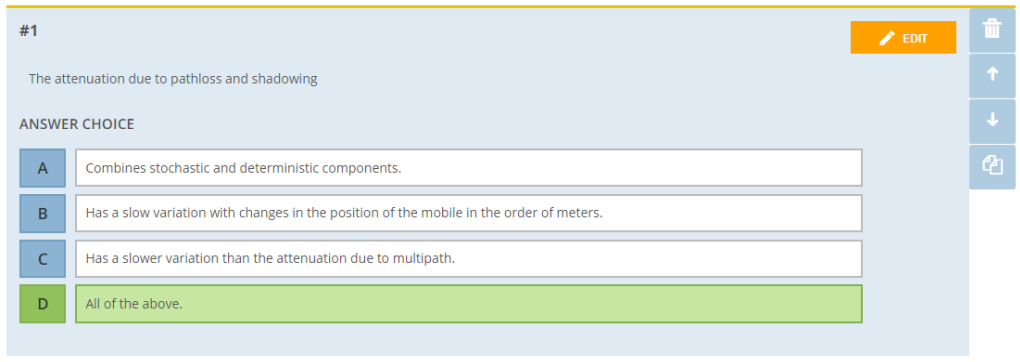

Fig. 1: Ejemplo de cuestionario de opción múltiple en modo simple.

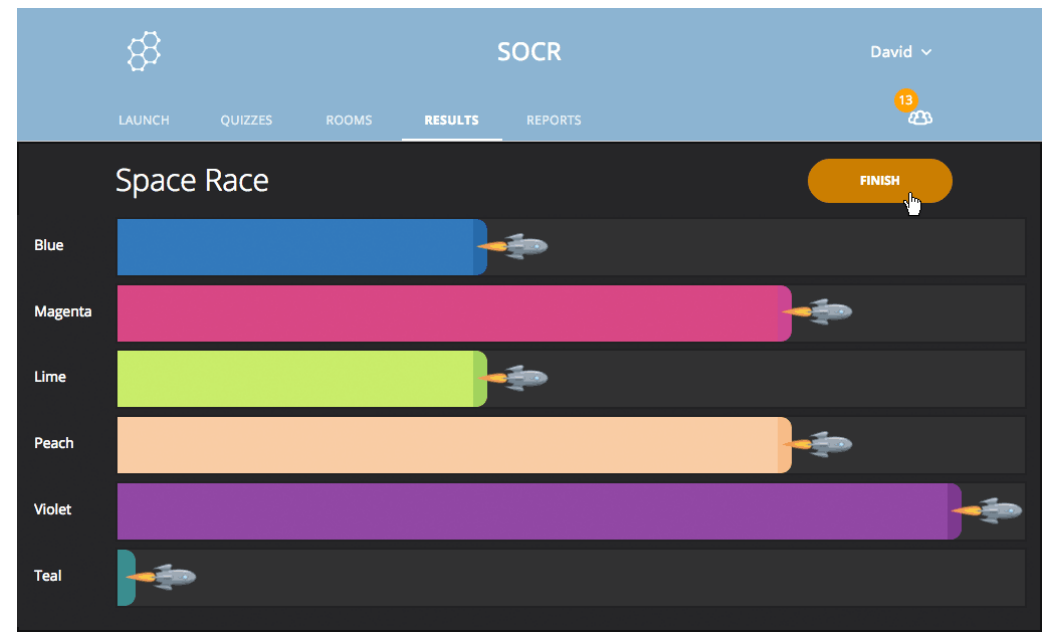

Fig. 2: Ejemplo de carrera espacial (modo gamificado).

\subsection{Consideraciones generales}

Algunas de las consideraciones previas tenidas en cuenta antes de la implementación práctica de actividades basadas en este tipo de herramientas son las siguientes:

- El profesor debe conocer la aplicación con suficiente profundidad antes de iniciar cualquier tipo de actividad.

- Es importante que los alumnos también conozcan la herramienta, por lo se deben realizar diversas pruebas antes de iniciar la actividad en sí.

- Dado que este tipo de herramientas funcionan únicamente bajo conexión a Internet, es necesario asegurar que tanto los alumnos como el profesor tienen un acceso adecuado a la red.

- Los estudiantes deben estar bien informados sobre lo que se espera de ellos, tanto en términos de la tecnología a utilizar como en reglas del juego.

- La gamificación debe utilizarse con moderación. Una sobreutilización puede arruinar el efecto deseado. 
- La variedad en las herramientas o modos de utilización ayuda a evitar el aburrimiento.

- Se debe recordar a los alumnos que la actividad debe servir para aprender y repasar conceptos a través de actividades de gamificación.

- Tal como ocurre en otras aplicaciones tecnológicas, a veces se producen diferencias en velocidad del acceso a la red o en las velocidades de los dispositivos que los alumnos utilizan. Controlar estos factores es importante.

\section{Metodología}

\subsection{Participantes}

El estudio se realizó sobre todos los alumnos y alumnas de dos asignaturas relacionadas con la Ingeniería de Telecomunicación. Por un lado, se consideró la asignatura Comunicaciones Móviles e Inalámbricas de $4^{\circ}$ curso del Grado de Ingeniería Telemática y, por otro, la asignatura Advanced Wireless Communications and Mobility del Máster de Ingeniería de Telecomunicación. Concretamente, la totalidad de alumnos que asistieron regularmente a clase en alguna de las dos asignaturas dio lugar a una muestra total de 25 estudiantes (12 de grado y 13 de máster).

\subsection{Procedimiento}

En todas las sesiones relativas al presente estudio, todo el alumnado contó con un dispositivo electrónico con conexión a internet (smartphone, tablet u ordenador portátil) para utilizar la aplicación gestora de participación a evaluar, es decir, Socrative.

En particular, el estudio se centró en el uso de los modos simple (cuestionario de opción múltiple) y modo gamificado (carrera espacial). Se realizaron cuestionarios de 10 preguntas con 4 posibles respuestas a elegir en 12 de las sesiones asociadas a cada una de las asignaturas. Cabe destacar que ambos modos no se utilizaron con la misma frecuencia, sino que el modo gamificado se consideró en solamente un $25 \%$ de las ocasiones frente a un $75 \%$ de veces en las que la opción fue el modo simple. Concretamente, al final de cada tema de la asignatura, se lanzó un cuestionario en modo simple, realizando en total 9 cuestionarios en este modo. Los cuestionarios gamificados se realizaban tras haber cubierto 3 temas diferentes y, por tanto, el contenido evaluado correspondía al de los tres cuestionarios de modo simple anteriores. Para motivar a los estudiantes a preparar los temas, se decidió dar el siguiente peso a los cuestionarios en la evaluación:

- La totalidad de los cuestionarios simples tenia un peso máximo de 1 punto adicional en la nota final de la asignatura. Por tanto, cada cuestionario simple tenía un peso máximo de $1 / 9$, que dependía del número de respuestas correctas del mismo.

- La totalidad de los tres cuestionarios gamificados también podía suponer un punto adicional en la nota final, de modo que cada carrera espacial valía 1/3. Sin embargo, para fomentar la competitividad en este modo, sólo el estudiante que 
ganara la carrera podía sumar esta cantidad, que era fija independientemente de que todas las respuestas hubieran sido correctas.

Los participantes fueron informados a principio de curso de la propuesta para incrementar su nota final anteriormente descrita y de las sesiones en las que se llevarían a cabo los cuestionarios.

\subsubsection{Encuestas}

Con el fin de estudiar la percepción del alumno respecto a la actividad propuesta, se realizó un breve cuestionario al final del curso (también mediante Socrative), en el cual aparecían los siguientes ítems a evaluar según una escala Likert de 5 niveles (1-Totalmente en desacuerdo, 2- En desacuerdo, 3- Ni de acuerdo ni en desacuerdo, 4-De acuerdo, 5- Totalmente de acuerdo):

- A - El modo Space Race me ha parecido más divertido que el modo normal.

- B - El modo Space Race me ha resultado más estresante que el modo normal.

- C - He dedicado más esfuerzo a preparar el modo Space Race que el normal.

- D - La actividad, en general, ha sido motivadora.

\section{Resultados del estudio}

Las Figuras 3 y 4 muestran los resultados de los 4 ítems evaluados en las encuestas de satisfacción que se realizaron a los alumnos de Grado y Máster, respectivamente. Las respuestas de los alumnos han sido representadas mediante histogramas, con una barra para cada puntuación, y donde la puntuación media se ha representado con una línea vertical roja.

En la Figura 3, se puede observar que la mayoría de alumnos de Grado ha encontrado el modo Space Race más divertido que el modo normal, a pesar de que también lo ha evaluado como más estresante. En cuanto al esfuerzo dedicado a preparar ambos tipos de cuestionarios, no hay un consenso entre los alumnos, ya que se observa bastante dispersión de puntuaciones en el histograma. Por último, cabe destacar que la mayoría del alumnado de grado ha encontrado la actividad en general motivadora. Los resultados mostrados en la Figura 4 muestran, a grandes rasgos, una opinión similar para los alumnos de Máster, aunque las puntuaciones medias son superiores a las de Grado para los 4 ítems. Se puede también observar que los alumnos de Máster han dado puntuaciones menos dispersas en las preguntas A y D.

Una vez discutidos los resultados de las encuestas, pasamos a analizar el rendimiento de los alumnos en los cuestionarios. La Figura 5 contiene el número de respuestas correctas en cada uno de los cuestionarios realizados en los distintos temas de la asignatura, tanto del tipo simple (un cuestionario por tema) como de tipo gamificado (un cuestionario cada tres temas). Las barras grises muestran los resultados del cuestionario simple y las barras amarillas los del gamificado. La media de respuestas correctas en cada cuestionario se ha representado mediante líneas horizontales continuas, en rojo para el tipo simple (MT_simple) y en verde para el tipo gamificado 

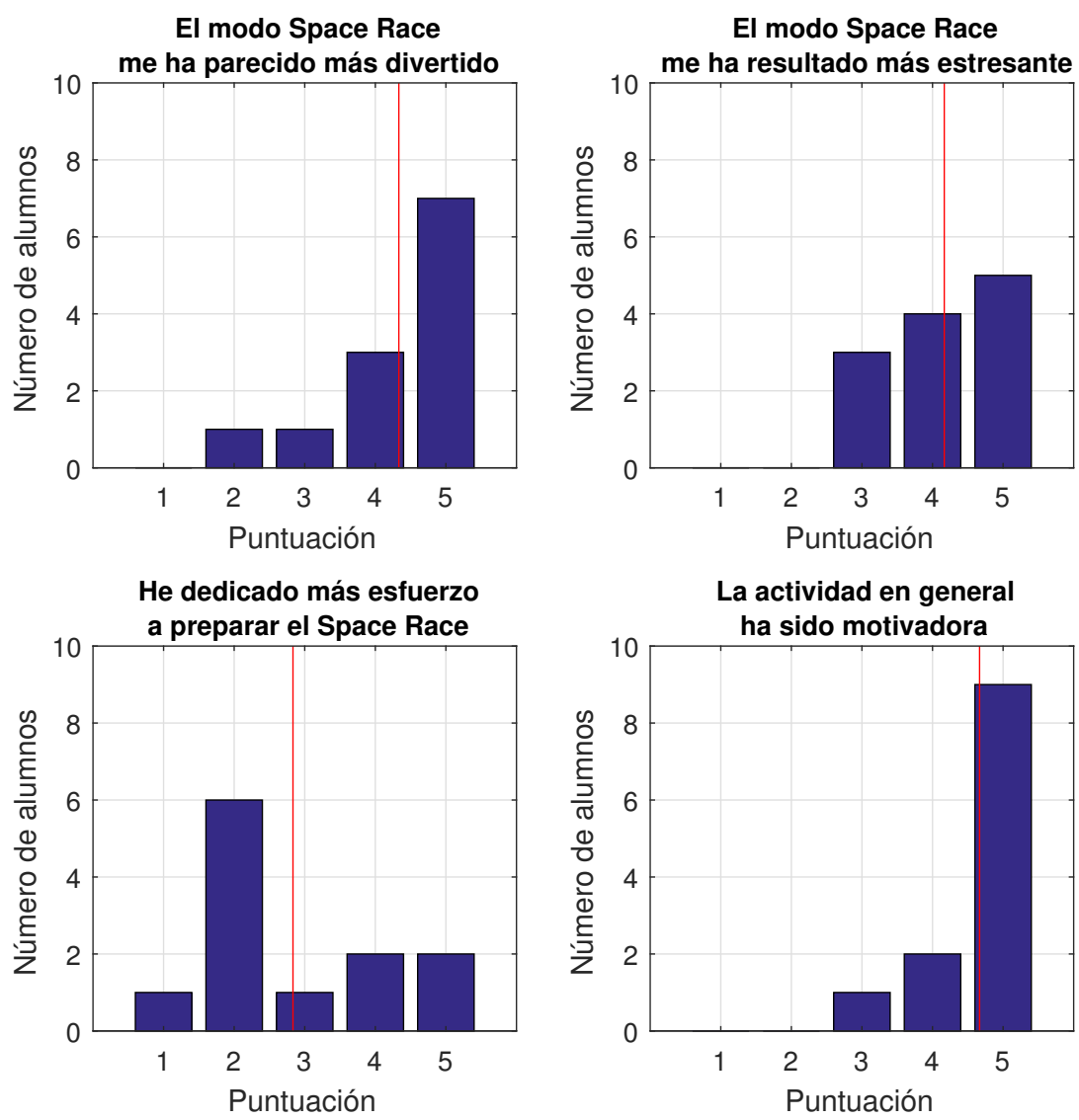

Fig. 3: Resultados de las encuestas de satisfacción para los alumnos de Grado.

(MT_gamificado). Se han utilizado también líneas punteadas horizontales para mostrar la media de respuestas correctas en el cuestionario simple en grupos de tres temas (M123, M456, M789). Esta última medida es útil para realizar una comparación justa con el número de respuestas correctas en cada cuestionario gamificado. La figura muestra resultados análogos para el caso de los alumnos de Máster.

En vista de los resultados, los alumnos de Grado han tenido un mejor rendimiento en los cuestionarios simples, siendo el número medio de respuestas correctas cerca de dos unidades mayor que en los gamificados (aproximadamente 5 frente a casi 7). Para el caso de los alumnos de Máster, también se ha observado el mismo efecto, pero la diferencia de rendimiento en ambos tipos de cuestionarios ha sido menor ( 5 respuestas correctas en gamificado frente a cerca de 6 en simple). En el caso de la asignatura de Grado, se puede observar una mejoría en los resultados a medida que los temas avanzan. Este hecho se puede extraer comparando las medias para los grupos de tres temas (M123 es menor que M456 y esta última menor a M789), lo que deja entrever que quizás los alumnos de grado se prepararon mejor los últimos temas que los primeros visto el funcionamiento y dificultad de los cuestionarios. En el caso del Máster, sin embargo, el número de respuestas correctas se ha mantenido 

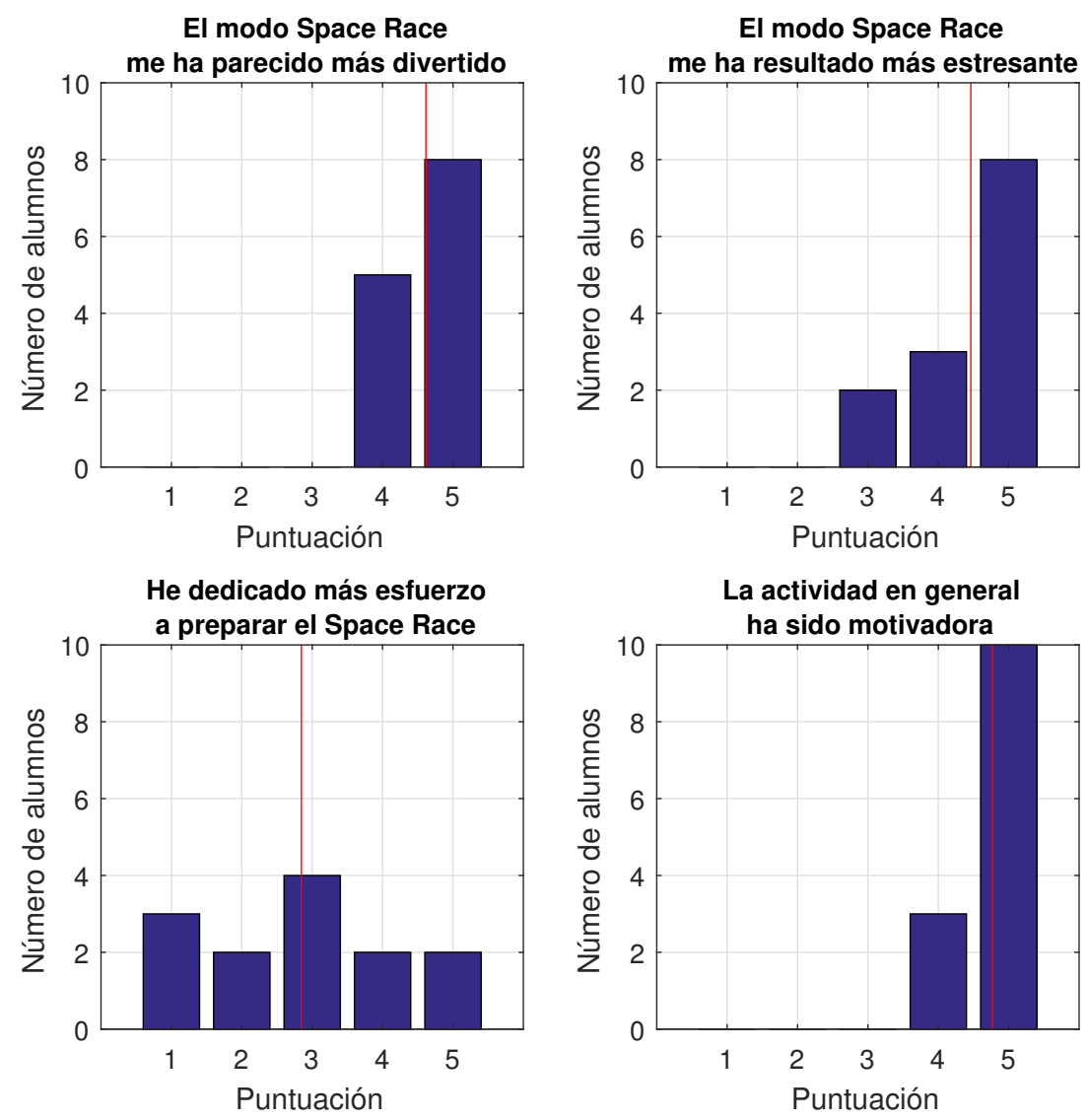

Fig. 4: Resultados de las encuestas de satisfacción para los alumnos de Máster.

bastante estable a lo largo del curso, siendo incluso inferior en media para los temas 7, 8 y 9. Por tanto, una hipótesis es que los alumnos de Máster se preparan por igual todos los cuestionarios, siendo los últimos de mayor dificultad, por ser partes de la asignatura de nivel más avanzado. Otro aspecto que puede influir en las diferencias observadas es el carácter optativo de la asignatura de Grado, lo cual podría explicar una mayor motivación por parte de los alumnos que la cursaban y, consecuentemente, unos mejores resultados en la misma.

Por último, la Figura 7 alberga la relación entre el esfuerzo subjetivo dedicado por los alumnos para preparar los cuestionarios de ambos tipos (según la puntuación reflejada en la pregunta $\mathrm{C}$ de las encuestas) y el número de respuestas correctas en cada uno de los cuestionarios. Nótese que para construir esta figura se han contabilizado solamente aquellas puntuaciones iguales a 4 y 5 como casos en los que el alumno empleó más esfuerzo en preparar el modo gamificado, y las puntuaciones iguales a 1 y 2 como casos en los que el alumno dedicó más esfuerzo en preparar el modo simple. Se ha prescindido del caso de puntuación 3, ya que implicaría que el alumno dedicó el mismo esfuerzo a ambos tipos de cuestionarios. Para los dos escenarios evaluados (Grado y Máster), se puede observar un número mayor de respuestas correctas en el modo 


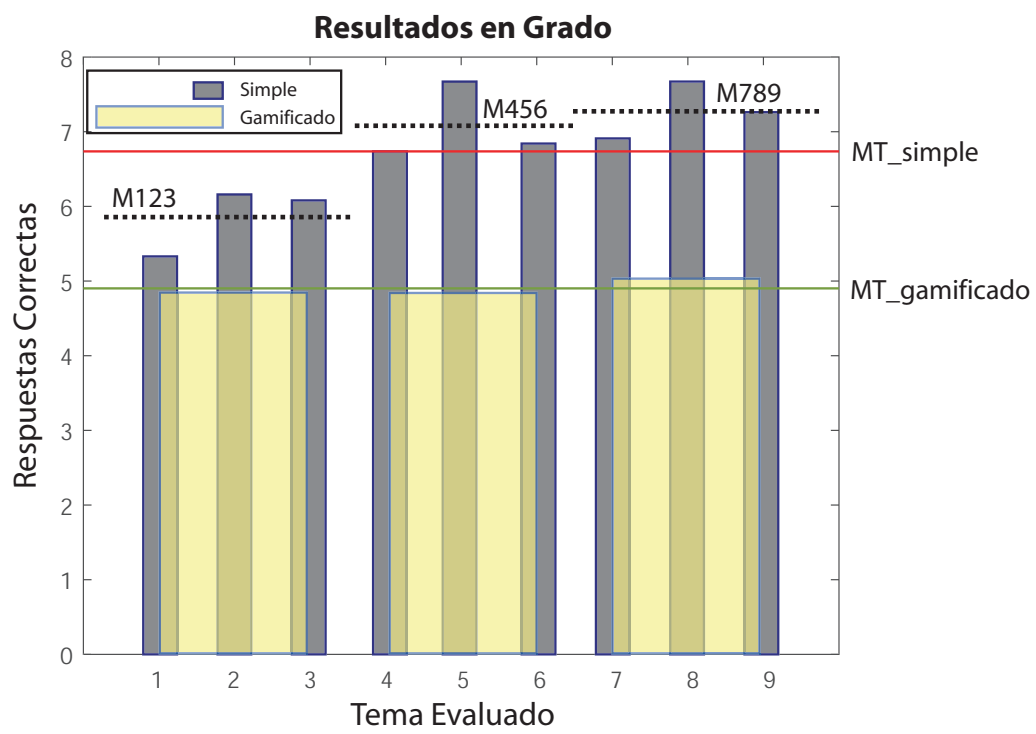

Fig. 5: Número medio de respuestas correctas en todos los cuestionarios realizados de ambos modos para los alumnos de Grado.

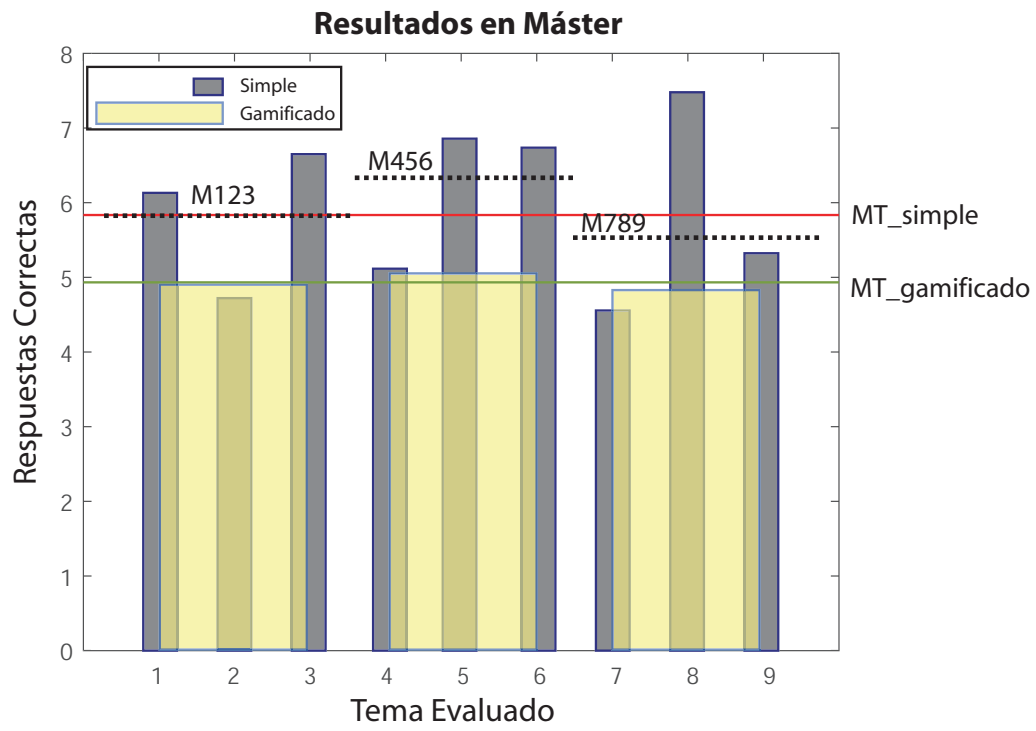

Fig. 6: Número medio de respuestas correctas en todos los cuestionarios realizados de ambos modos para los alumnos de Máster.

simple, independientemente de que se trate de alumnos que hayan preparado más este tipo de cuestionario o el gamificado. Por tanto, en general se puede concluir que el tipo de cuestionario gamificado supuso una mayor dificultad para el alumnado, algo que ya dejaban entrever las figuras 5 y 6 . De hecho, los resultados obtenidos en los cuestionarios gamificados fueron en media algo mayores para aquellos alumnos que habían dedicado más esfuerzo a la preparación de los cuestionarios simples, tanto en la asignatura de Grado como en la de Máster. Esta observación hace suponer que los alumnos que habían preparado mejor los cuestionarios simples de una forma 
continuada a lo largo del curso también lo hacían mejor en el modo gamificado, por lo que probablemente afianzaron mejor sus conocimientos.

\section{Conclusiones}

El presente artículo se ha centrado en la realización de cuestionarios al alumnado combinando los modos simple y de carrera espacial del gestor de participación Socrative. El estudio del uso de ambos modos se ha realizado sobre dos asignaturas relacionadas con la Ingeniería de Telecomunicación, una de nivel de Grado y otra de Máster. Se ha podido observar, tras realizar encuestas de opinión a los participantes, que el uso de Socrative para realizar cuestionarios resulta muy motivador, habiendo mostrado los alumnos preferencia por los de tipo gamificado (carrera espacial). En cuanto al rendimiento de los alumnos en los cuestionarios de ambos tipos, los resultados indican un mayor número de respuestas acertadas con los cuestionarios de tipo simple. Por tanto, la preferencia por los cuestionarios de tipo gamificado no implica una mejora en la nota obtenida. Cabe remarcar que los resultados obtenidos en los cuestionarios gamificados fueron en media algo mejores para aquellos alumnos que habían dedicado más esfuerzo a la preparación de los cuestionarios simples, tanto en la asignatura de Grado como en la de Máster. A raíz de los resultados se puede concluir que los alumnos que habían preparado mejor los cuestionarios simples de una forma continuada a lo largo del curso también lo hubieran hecho mejor para los del modo gamificado, afianzando así mejor sus conocimientos.

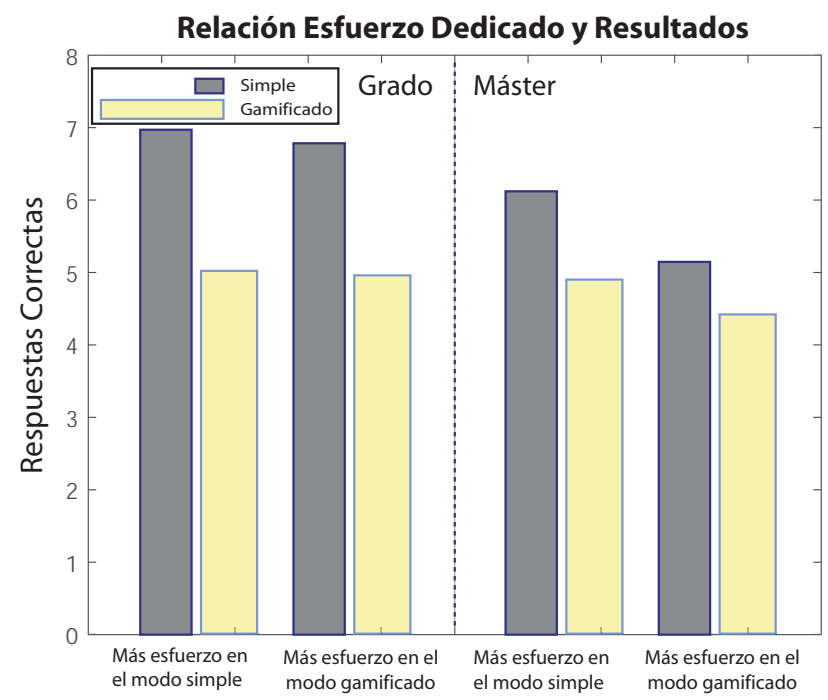

Fig. 7: Relación entre el esfuerzo dedicado por el alumnado para cada tipo de cuestionario y sus resultados medios obtenidos. 
Combinación de cuestionarios simples y gamificados utilizando gestores de participación en el aula: experiencia y percepción del alumnado

\section{Referencias bibliográficas}

Benítez-Porres, J. (2015). "Socrative como herramienta para la integración de contenidos en la asignatura "Didáctica de los Deportes"". En: XII Jornadas Internacionales de Innovación Universitaria Educar para transformar: Aprendizaje experiencial, Madrid, Julio 2015, págs. 824-831.

Calvillo, A.J. y D. Martín (2017). The Flipped Learning: Guía "gamificada"para novatos y no tan novatos. Universidad Internacional de La Rioja (UNIR Editorial).

Eisele-Dyrli, K. (2011). "Mobile Goes Mainstream". En: District Administration 47.2, págs. 46-55.

Frías, M.V., C. Arce y P. Flores-Morales (2016). "Uso de la plataforma socrative.com para alumnos de Química General". En: Educación Química 27.1, págs. 59-66.

Fuertes, A. y col. (2016). "Uso de herramientas de respuesta de audiencia en la docencia presencial universitaria. Un primer contacto." En: XXII Jornadas sobre la Enseñanza Universitaria de la Informática (JENUI 2016), Almería, Spain, Julio 2016, págs. 257-265.

Kahoot. https://kahoot.it/.

Kay, R. y A. LeSage (2009). "A strategic assessment of audience response systems used in higher education". En: Australasian Journal of Educational Technology 25.2, págs. 235-249.

Melhuish, K. y G. Falloon (2010). "Looking to the future: M-learning with the iPad Computers in New Zealand Schools". En: Learning, Leading, Technology 22.3, págs. 1-16.

Socrative. https://www. socrative.com. 